OPEN ACCESS

Check for updates

\title{
Comparative efficacy and safety of new surgical treatments for benign prostatic hyperplasia: systematic review and network meta-analysis
}

\author{
Shi-Wei Huang, ${ }^{1}$ Chung-You Tsai, ${ }^{2}$ Chi-Shin Tseng, ${ }^{3}$ Ming-Chieh Shih,,${ }^{4}$ Yi-Chun Yeh, \\ Kuo-Liong Chien, ${ }^{4}$ Yeong-Shiau Pu, ${ }^{3}$ Yu-Kang Tu ${ }^{4,5}$
}

${ }^{1}$ Department of Urology,

National Taiwan University Hospital Yunlin Branch, Yunlin County, Taiwan

${ }^{2}$ Division of Urology,

Department of Surgery, Far

Eastern Memorial Hospital, New

Taipei City, Taiwan

${ }^{3}$ Department of Urology,

National Taiwan University

Hospital, Taipei, Taiwan

${ }^{4}$ Institute of Epidemiology and

Preventive Medicine, National

Taiwan University, 17 Hsu-Chow

Road, Taipei, Taiwan

${ }^{5}$ Department of Medical

Research, National Taiwan

University Hospital, Taipei,

Taiwan

Correspondence to: $\mathrm{Y}-\mathrm{K} T \mathrm{U}$

yukangtu@ntu.edu.tw

(ORCID 0000-0002-2461-474X)

Additional material is published online only. To view please visit the journal online.

Cite this as: BMJ 2019:367:15919 http://dx.doi.org/10.1136/bmj.l.5919

Accepted: 27 September 2019

\section{ABSTRACT}

OBJECTIVE

To assess the efficacy and safety of different endoscopic surgical treatments for benign prostatic hyperplasia.

\section{DESIGN}

Systematic review and network meta-analysis of randomised controlled trials.

\section{DATA SOURCES}

A comprehensive search of PubMed, Embase, and Cochrane databases from inception to 31 March 2019.

\section{STUDY SELECTION}

Randomised controlled trials comparing vapourisation, resection, and enucleation of the prostate using monopolar, bipolar, or various laser systems (holmium, thulium, potassium titanyl phosphate, or diode) as surgical treatments for benign prostatic hyperplasia. The primary outcomes were the maximal flow rate (Qmax) and international prostate symptoms score (IPSS) at 12 months after surgical treatment. Secondary outcomes were Qmax and IPSS values at 6, 24, and 36 months after surgical treatment; perioperative parameters; and surgical complications.

\section{DATA EXTRACTION AND SYNTHESIS}

Two independent reviewers extracted the study data and performed quality assessments using the Cochrane Risk of Bias Tool. The effect sizes were summarised using weighted mean differences for continuous outcomes and odds ratios for binary outcomes. Frequentist approach to the network metaanalysis was used to estimate comparative effects and safety. Ranking probabilities of each treatment were also calculated.

\section{WHAT IS ALREADY KNOWN ON THIS TOPIC}

Monopolar transurethral resection of the prostate has long been the standard treatment of benign prostatic hyperplasia

Many new energy systems (eg, bipolar electrodes, or thulium, holmium, diode, or potassium titanyl phosphate lasers) emerged in 2000 , and have been used for transurethral treatment of benign prostatic hyperplasia

\section{WHAT THIS STUDY ADDS}

All endoscopic enucleation methods (including bipolar electrodes, or holmium, thulium, or diode lasers) showed better functional outcomes than vapourisation and resection methods

Eight new surgical methods using bipolar electrode or laser treatments were superior in controlling bleeding (intraoperatively and postoperatively) compared with monopolar transurethral resection of the prostate

\section{RESULTS}

109 trials with a total of 13676 participants were identified. Nine surgical treatments were evaluated. Enucleation achieved better Qmax and IPSS values than resection and vapourisation methods at six and 12 months after surgical treatment, and the difference maintained up to 24 and 36 months after surgical treatment. For Qmax at 12 months after surgical treatment, the best three methods compared with monopolar transurethral resection of the prostate (TURP) were bipolar enucleation (mean difference 2.42 $\mathrm{mL} / \mathrm{s}$ (95\% confidence interval 1.11 to 3.73 )), diode laser enucleation (1.86 (-0.17 to 3.88)), and holmium laser enucleation (1.07 (0.07 to 2.08)). The worst performing method was diode laser vapourisation $(-1.90(-5.07$ to 1.27$))$. The results of IPSS at 12 months after treatment were similar to Qmax at 12 months after treatment. The best three methods, versus monopolar TURP, were diode laser enucleation (mean difference -1.00 ( -2.41 to 0.40$)$ ), bipolar enucleation (0.87 ( -1.80 to 0.07$)$ ), and holmium laser enucleation ( $-0.84(-1.51$ to 0.58$)$ ). The worst performing method was diode laser vapourisation (1.30 ( -1.16 to 3.76)). Eight new methods were better at controlling bleeding than monopolar TURP, resulting in a shorter catheterisation duration, reduced postoperative haemoglobin declination, fewer clot retention events, and lower blood transfusion rate. However, short term transient urinary incontinence might still be a concern for enucleation methods, compared with resection methods (odds ratio 1.92, 1.39 to 2.65 ). No substantial inconsistency between direct and indirect evidence was detected in primary or secondary outcomes.

\section{CONCLUSION}

Eight new endoscopic surgical methods for benign prostatic hyperplasia appeared to be superior in safety compared with monopolar TURP. Among these new treatments, enucleation methods showed better Qmax and IPSS values than vapourisation and resection methods.

STUDY REGISTRATION

CRD42018099583.

\section{Introduction}

Lower urinary tract symptoms caused by benign prostatic hyperplasia are the most common urological problem among men, affecting about a third of men over age $50 .^{12}$ Surgical intervention is the most effective treatment for benign prostatic hyperplasia, with around 100000 procedures carried out annually 
in the United States. ${ }^{3}$ Of all surgical treatments, monopolar transurethral resection of the prostate (TURP), in which the enlarged prostate tissue is resected piece by piece using a monopolar electrode, has been the preferred method since the 1970s. It can substantially improve the maximal flow rate (Qmax), urinary symptoms (based on the international prostate symptom score (IPSS)), and health related quality of life, with long term efficacy compared with drugs or other minimally invasive treatments. ${ }^{45}$ However, monopolar TURP is a risky procedure because of the likelihood of severe complications such as massive bleeding or transurethral resection syndrome. ${ }^{6}$ Therefore, minimally invasive surgical techniques need to be developed with outcomes similar to those of monopolar TURP, but with fewer side effects. ${ }^{5}$

Since the 2000s, new energy systems for surgical interventions treating benign prostatic hyperplasia have quickly become popular, including systems that use bipolar energy and various laser systems such as the holmium laser, potassium titanyl phosphate (KTP) laser, thulium laser, and diode laser. ${ }^{7} 8$ The trend in surgical treatment for benign prostatic hyperplasia has shifted from monopolar TURP to laser treatments and bipolar TURP over the past 10 years. ${ }^{3}$ Bipolar energy can be used to incise, resect, and vapourise prostate tissue using different electrodes. Holmium and thulium laser beams are mainly absorbed by water and act as incisional lasers. The KTP laser is selectively absorbed by haemoglobin and debulks prostate tissue through vapourisation. The diode laser is absorbed by water and haemoglobin can therefore vapourise and incise prostate tissue. These new methods all use normal saline instead of distilled water to avoid hyponatraemia.

The new methods can be further divided into three types according to their treatment principles: resection methods (resection of prostate tissue piece by piece), vapourisation methods (vapourisation of excessive prostate tissue), and enucleation methods (peeling the enlarged prostate from the prostate capsule). Table 1 lists the nomenclature and abbreviations of the nine surgical methods and figure 1 illustrates the description of the surgical methods. These new methods are intended to replace monopolar TURP, which is the standard surgical treatment for benign prostatic hyperplasia.

The aim of this systematic review and network metaanalysis was to investigate the new surgical methods and determine which achieves the best functional outcomes with fewer complications by evaluating data from published randomised controlled trials.

\section{Methods}

\section{Search strategy and selection criteria}

This study followed PRISMA recommendations (preferred reporting items for systematic reviews and meta-analyses). ${ }^{9}$ The method and analysis were prespecified in advance and registered on the PROSPERO website (CRD42018099583). To identify published and unpublished trials, we used electronic databases including PubMed (inception to March 2019), Embase (inception to March 2019), and Cochrane clinical trial registers (inception to March 2019) without language or date restriction, as well as performing a manual literature search. The detailed study protocol including search terms and strategy is provided in the supplementary material and supplementary table 1 . Randomised parallel group design clinical trials comparing any two of the different surgical methods were eligible for inclusion. All methods are listed in table 1. Inclusion criteria were patients with a Qmax lower than $15 \mathrm{~mL} / \mathrm{s}$ and an IPSS greater than 8. Exclusion criteria were patients with neurogenic bladder; previous urethral, prostate, or bladder surgeries; and suspected prostate cancer.

\section{Outcome measures}

The outcome measures for the analysis included:

- Functional outcomes: Qmax and IPSS at 6, 12, 24, and 36 months after surgical treatment

- Perioperative parameters: catheterisation duration and haemoglobin declination

- Short term complications including transurethral resection syndrome, clot retention (blood clot retention in the bladder), blood transfusion, urinary tract infection, recatheterisation, and incontinence

- Long term complications including urinary strictures, retrograde ejaculation, and recurrence (recurrence of benign prostatic hyperplasia requiring reoperation or repeat apical resection).

Long term complications were only included if the data were from trials with more than three months' follow-up. We chose the Qmax and IPSS at 12 months after surgical treatment as primary outcomes and other clinical measurements as secondary outcomes.

\section{Data extraction and quality assessment}

Two reviewers (S-WH, C-ST) independently screened the titles and abstracts for eligibility. The full articles were then assessed regarding eligibility criteria. We developed a data extraction form, which we pilot tested in 10 randomly included studies, and then refined it accordingly. Two reviewers (S-WH, C-ST) extracted data independently and then cross checked the data. We used the Cochrane Collaboration's Risk of Bias tool to appraise study quality. ${ }^{10}$ Any unresolved discrepancies in data extraction or appraisal of the results were evaluated by a third reviewer (C-YT) who acted as an arbiter. We further applied the GRADE approach (grading of recommendation, assessment, development, and evaluation) to assess evidence quality regarding the primary outcomes, which was considered to be critical in clinical decision making. ${ }^{11}$

We attempted to contact some authors about missing data, and several authors responded. When standard deviation data were missing, or only the values before treatment were available, we calculated standard deviations with formulas described in the Cochrane handbook for systematic reviews of interventions ${ }^{10}$ or 


\begin{tabular}{|c|c|c|c|}
\hline $\begin{array}{l}\text { Surgical technique } \\
\text { and abbreviation }\end{array}$ & Surgical method (full terminology) & Energy source & Abbreviation \\
\hline \multicolumn{4}{|l|}{ Resection } \\
\hline Monopolar TURP & Monopolar transurethral resection of prostate & Monopolar & M-TURP \\
\hline Bipolar TURP & Bipolar transurethral resection of prostate & Bipolar & B-TURP \\
\hline \multicolumn{4}{|l|}{ Enucleation } \\
\hline Thulium LEP & Thulium laser enucleation of prostate & Thulium laser & ThuLEP \\
\hline Holmium LEP & Holmium laser enucleation of prostate & Holmium laser & HoLEP \\
\hline Diode LEP & Diode laser enucleation of prostate & Diode laser & DioLEP \\
\hline Bipolar EP & Bipolar enucleation of prostate & Bipolar & Bipolar TUEP \\
\hline \multicolumn{4}{|l|}{ Vapourisation } \\
\hline Diode LVP & Diode laser vapourisation of prostate & Diode laser & DioLVP \\
\hline KTP LVP & Potassium titanyl phosphate laser vapourisation of prostate & KTP laser & PVP, Greenlight \\
\hline Bipolar VP & Bipolar vapourisation of prostate & Bipolar & Bipolar TUVP \\
\hline
\end{tabular}

calculated it from the articles' figure data. If the authors only reported medians, we used medians as means and interquartile ranges/1.35 as the standard deviations. ${ }^{12}$

\section{Statistical methods}

We conducted a pairwise random effect metaanalysis. The weighted mean differences and odds ratios were reported for continuous and binary variables, respectively. Heterogeneity was assessed by visual inspection of the forest plot and tested using $\mathrm{I}^{2}$ statistics. ${ }^{10}$ For continuous data, we used the Dersimonian and Laird method; for binary variables, we conducted a one stage meta-analysis using a generalised linear mixed model with the exact binomial likelihood.

Next, we undertook a frequentist network metaanalysis for each outcome separately. For continuous variables, such as functional outcomes and perioperative parameters, we performed a contrast based network meta-analysis using Stata (version 14, Stata, College Station, TX) through a network module based on the mvmeta command for multiple treatment comparisons with the restricted maximum likelihood approach. ${ }^{13}$ Variances between studies were equalised, correlations were set to 0.5 , and confidence intervals were estimated on the basis of asymptotic error variance and normal distribution.

For dichotomous variables such as complications, we noted rare and zero events. Trials with zero events in all arms of each outcome were deleted during the analysis because they offered no valuable information. We conducted the arm based network meta-analysis using generalised linear mixed models ${ }^{14}$ with a restricted maximum likelihood approach. No imputation for zero cell counts was performed. All analyses for binary data were undertaken using the GLIMMIX procedure of the SAS software version 9.4 (SAS Institute, Cary, NC) with the Laplace integration method.

We evaluated potential inconsistencies between direct and indirect evidence within the network metaanalysis using the design-by-treatment interaction model $^{15}$ and the side splitting method. ${ }^{16}$ The designby-treatment interaction model provides a global assessment of consistency across the entire network. The side splitting method separated evidence on a particular comparison into direct and indirect evidence and then assessed their differences. Statistical significance was set at $5 \%$ for all analyses.

We also estimated the probabilities of each treatment being at each rank for each intervention and outcome. We obtained a treatment hierarchy using the surface under the cumulative ranking curve and mean ranks. ${ }^{17}$

\section{Sensitivity analysis}

Prostate size could affect the outcomes of the different surgical treatment methods-that is, large prostates might be better suited to treatment via enucleation methods, and less effectively treated using vapourisation methods. We performed a metaregression analysis according to the mean prostate volume data provided in each trial report. To increase the power of the meta-regression, assuming that the functional outcomes would be similar with similar surgical techniques, we grouped the nine methods into four types: enucleation, vapourisation, bipolar TURP, and monopolar TURP. We defined the large prostate group as having a mean prostate volume of more than $70 \mathrm{~mL}$ and also undertook an analysis with a cutoff value of $60 \mathrm{~mL}$. We also compared mean differences in Qmax and IPSS between these four types of surgical methods at 6, 12, 24, and 36 months after surgical treatment. We further compared short term transient incontinence ( $<1$ month after surgical treatment) and permanent incontinence rate ( $>6-12$ months after surgical treatment) between enucleation (excluding vapo-enucleation) and resection methods.

\section{Patient and public involvement}

This research was done without patient involvement. Patients were not invited to comment on the study design and were not consulted to develop patient relevant outcomes or interpret the results. Patients were not invited to contribute to the writing or editing of this document for readability or accuracy. Results of studies are to be disseminated to patients and patient groups through social media.

\section{Results}

The flowchart in supplementary figure 1 shows the literature search process to obtain eligible trials. We identified 1821, 3469, and 241 articles from PubMed, Embase, and the Cochrane clinical trials, 


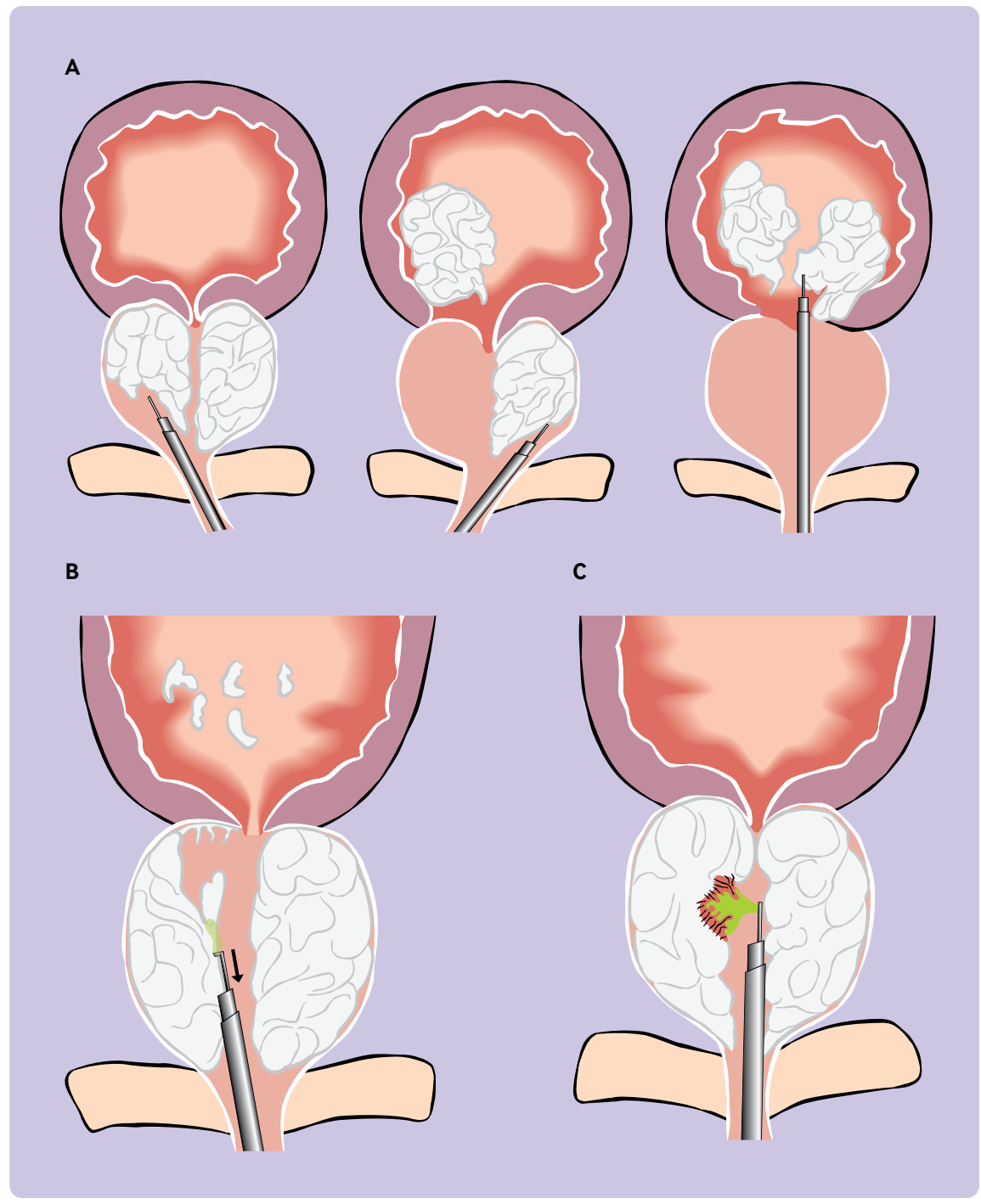

Fig 1 | Different endoscopic surgical methods for benign prostate hyperplasia. (A) Enucleation methods: peeling the entire prostate adenoma from the prostate capsule using end-firing laser fibre or designed bipolar loop, then morcellating the adenoma with a shaver (B) Resection methods: resecting the enlarged prostate adenoma with monopolar or bipolar resection loop, piece by piece. (C) Vapourisation methods: vapourising the enlarged prostate adenoma with side-firing laser fibre or mushroom-like bipolar electrode

respectively. After eliminating 1564 duplicate articles, the total number of articles was 3967. Of those, 3744 articles were excluded on the basis of the abstract and title reviews. Of the remaining 223 articles with the full texts reviewed, 136 articles in 109 trials met our inclusion criteria for the systematic review and meta-analysis. The 109 eligible trials enrolled a total of 13676 participants and evaluated nine different surgical treatments for benign prostatic hyperplasia with 21 direct comparisons.

Among those 109 trials, three had three arms and 106 had two arms; most comparisons included bipolar TURP, bipolar VP, holmium LEP, and KTP LVP with monopolar TURP methods (table 1; fig 2). The clinical and methodological characteristics and the studied outcomes of each trial are summarised in supplementary tables 2-4. Baseline characteristics including age, preoperative IPSS, Qmax, and quality of life were similar among all trials; however, mean prostate volume was not. The medians and interquartile ranges for age, Qmax, IPSS, and quality of life were 67.8 (4.3), 7.2 (1.9), 23.3 (2.8), and 4.50 (0.60), respectively. Among 101 trials that provided preoperative mean prostate volume data, eight, 74 , and 19 trials showed mean prostate volumes of up to 40, 40-70, and more than $70 \mathrm{~mL}$, respectively.

The Cochrane Collaboration's Risk of Bias assessment is shown in supplementary figure 2. High risk of bias was rare in any domain. However, unclear assessments were common, because some articles did not describe the randomisation methods, or whether the participants or outcome assessors were blinded. Regarding selective reporting, only $33 \%$ of trials $(n=36)$ were judged as having low risk of bias in reporting complications because they used the modified Clavien-Dindo classification ${ }^{18}$ or reported complications in detail. 


\section{All treatments comparison}

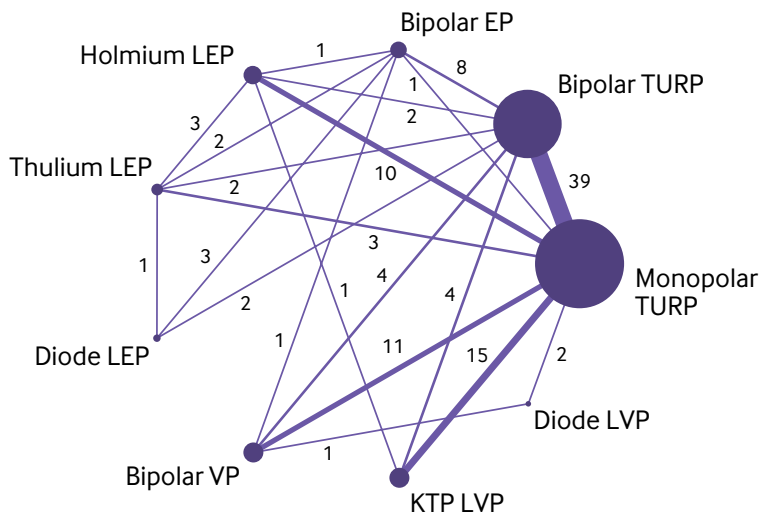

Qmax 6 months after treatment

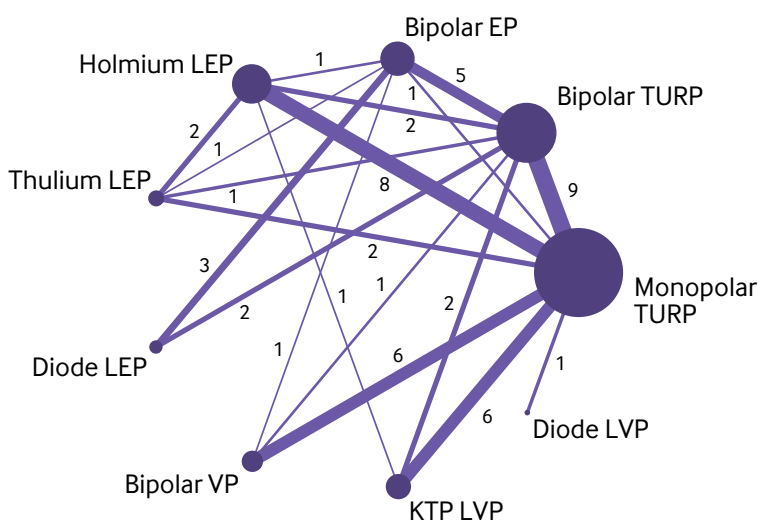

Qmax 12 months after treatment

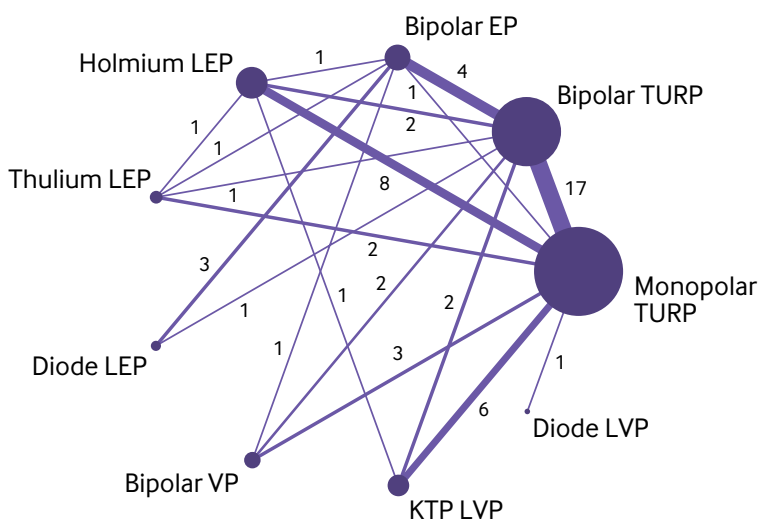

Fig 2 | Network treatment comparisons for all studies investigating new surgical methods for benign prostate hyperplasia. Figure shows treatment comparisons (all included studies, irrespective of the outcomes), Qmax values at six months after surgical treatment, and Qmax values at 12 months after surgical treatment. Node size corresponds to the number of trials in which the treatments were studied; interventions that are compared directly are joined with a line, the thickness of which corresponds to the number of trials that assessed the comparisons; the number of trials is shown on the line. Abbreviations of surgical methods are listed in table 1

\section{Functional outcomes}

A network of eligible comparisons for the primary outcome is presented in figure 2 and supplementary figure 3 . In the analysis, 51, 54, 18, and 14 trials reported Qmax values at 6, 12, 24, and 36 months after surgical treatment, respectively; corresponding numbers for IPSS values were 51, 53, 17, and 14 trials, respectively. These included predominantly pairwise comparisons of bipolar TURP, bipolar VP, holmium LEP, and KTP LVP with monopolar TURP for Qmax and IPSS values at six and 12 months after surgical treatment. Outcomes for the postsurgical follow-up at 24-36 months were only available for seven of the nine surgical methods compared in our network metaanalysis. Most trials performed a pairwise comparison between bipolar and monopolar TURP or compared holmium LEP and KTP LVP with bipolar or monopolar TURP.

We summarised our random effects network metaanalysis and pairwise comparison of functional outcomes in supplementary tables 5 and 7 . We ranked the comparative effects of eight new methods against monopolar TURP and the SUCRA probability (fig 3 and fig 4; supplementary table 6 and supplementary fig 5).

The four enucleation methods ranked highly, followed by the resection and vapourisation methods with respect to Qmax values at six and 12 month followup (fig 3). Mean differences in Qmax values ranged from $2.67 \mathrm{~mL} / \mathrm{s}$ (95\% confidence interval 0.96 to 4.39) for the highest ranked treatment (bipolar EP) to -0.68 $\mathrm{mL} / \mathrm{s}(-2.37$ to 1.00$)$ for the lowest ranked treatment (KTP LVP) at six months after surgical treatment; and from $2.42 \mathrm{~mL} / \mathrm{s}$ (1.11 to 3.73) for the highest ranked treatment (bipolar EP) to $-1.90 \mathrm{~mL} / \mathrm{s}(-5.07$ to 1.27$)$ for the lowest ranked treatment (diode LVP) at 12 months after surgical treatment. For the Qmax value at 12 months after surgical treatment, some treatments (bipolar EP, holmium LEP, and bipolar TURP) reached statistical significance when compared with monopolar TURP. Significant differences and rankings were similar at 24 and 36 months after surgical treatment.

The enucleation methods also ranked higher than the resection and vapourisation methods for IPSS values at six and 12 months after surgical treatment (fig 3). The mean difference in IPSS values compared with monopolar TURP ranged from -0.62 (95\% confidence interval -1.76 to 0.51$)$ for the highest ranked treatment (bipolar EP) to $0.70(-2.26$ to 3.66) for the lowest ranked treatment (diode LVP), at six months after surgical treatment; and from -1.00 $(-2.41$ to 0.40$)$ for the highest ranked treatment (diode LEP) to $1.30(-1.16$ to 3.76$)$ for the lowest ranked treatment (diode LVP), at 12 months after surgical treatment.

\section{Perioperative parameters}

The duration of catheterisation was reported in 82 trials. All methods using laser energy (diode, thulium, holmium, KTP) were ranked higher, followed by bipolar energy, but all performed better than monopolar TURP. Compared with monopolar TURP, catheterisation duration decreased from 43.07 hours (95\% confidence interval 29.96 to 56.17 ) for diode LEP to 10.80 hours (6.15 to 15.44) for bipolar TURP (fig 4).

Haemoglobin declination was reported in 68 trials; vapourisation and enucleation methods were ranked higher than bipolar TURP, and all performed better than monopolar TURP. Compared with monopolar 


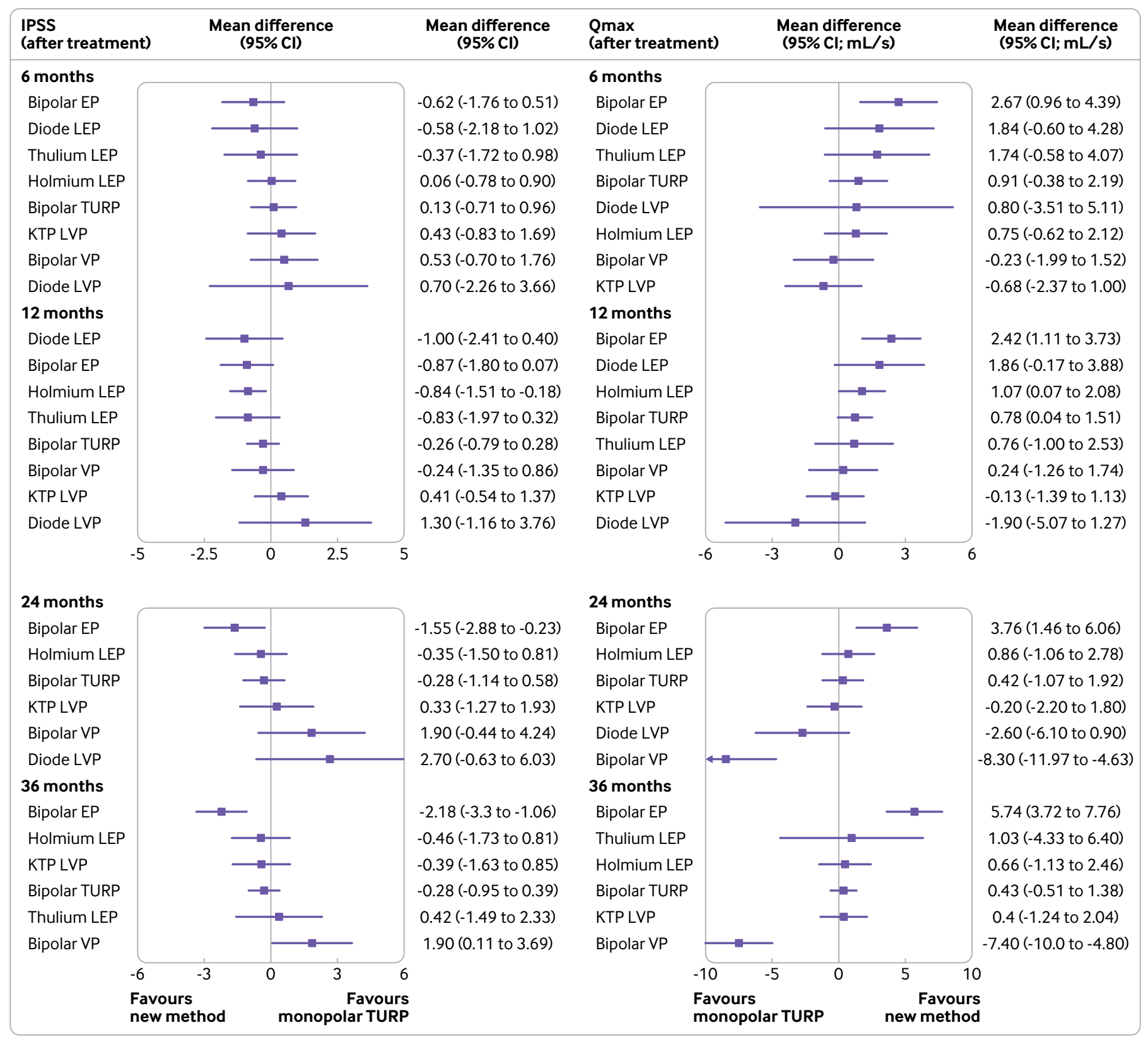

Fig 3 | Network meta-analysis of functional outcomes of new surgical methods compared with monopolar transurethral resection of the prostate (TURP) for benign prostate hyperplasia. Common heterogeneity variables for all comparisons in this network meta-analysis included: $\tau=1.99$, $1.13,1.05$, and 0.72 for $Q \max$ values at 6, 12, 24, and 36 months after surgical treatment, respectively, and $\tau=1.60,1.08,0.98$, and 0.75 for IPSS values $6,12,24$, and 36 months after surgical treatment, respectively. Treatments ranked according to the SUCRA values. SUCRA=surface under the cumulative ranking. Abbreviations of surgical methods are listed in table 1

TURP, the decline in haemoglobin decreased from $12.5 \mathrm{~g} / \mathrm{L}$ (8.4 to 16.6) for the highest ranked method (KTP LVP) to $1.9 \mathrm{~g} / \mathrm{L}$ (0.1 to 3.8) for the lowest ranked method (bipolar TURP; fig 4).

\section{Complications}

We analysed short term complications, including transurethral resection syndrome, recatheterisation, clot retention, blood transfusion, and incontinence, and long term related complications, including recurrence, urethral stricture, and retrograde ejaculation. The results of the network meta-analysis and pairwise comparison are shown in supplementary table 7 and supplementary figure 6. These adverse events were sparse and even zero in some trials; therefore, some interventions were lacking data for comparisons.

Regarding transurethral resection syndrome, no events were reported in the new methods. Clot retention and blood transfusion events were reported in 57 and 88 trials, respectively. Vapourisation and enucleation methods using either laser or bipolar energy were ranked higher than bipolar TURP, and all performed better than monopolar TURP. Compared 
with monopolar TURP, the odds ratio ranged from 0.12 (95\% confidence interval; 0.02 to 0.76 ) for bipolar EP to 0.49 (0.32 to 0.74 ) for bipolar TURP regarding clot retention, and from 0.05 (0.01 to 0.22) for holmium LEP to 0.42 (0.28 to 0.61 ) for bipolar TURP regarding blood transfusion.
In the 71 trials reporting recatheterisation events, enucleation methods ranked higher than resection methods, while vapourisation methods showed the worst outcomes. Compared with monopolar TURP, the odds ratio ranged from 0.27 (95\% confidence interval 0.11 to 0.69 ) for bipolar EP to 2.17 (0.34 to 13.9)

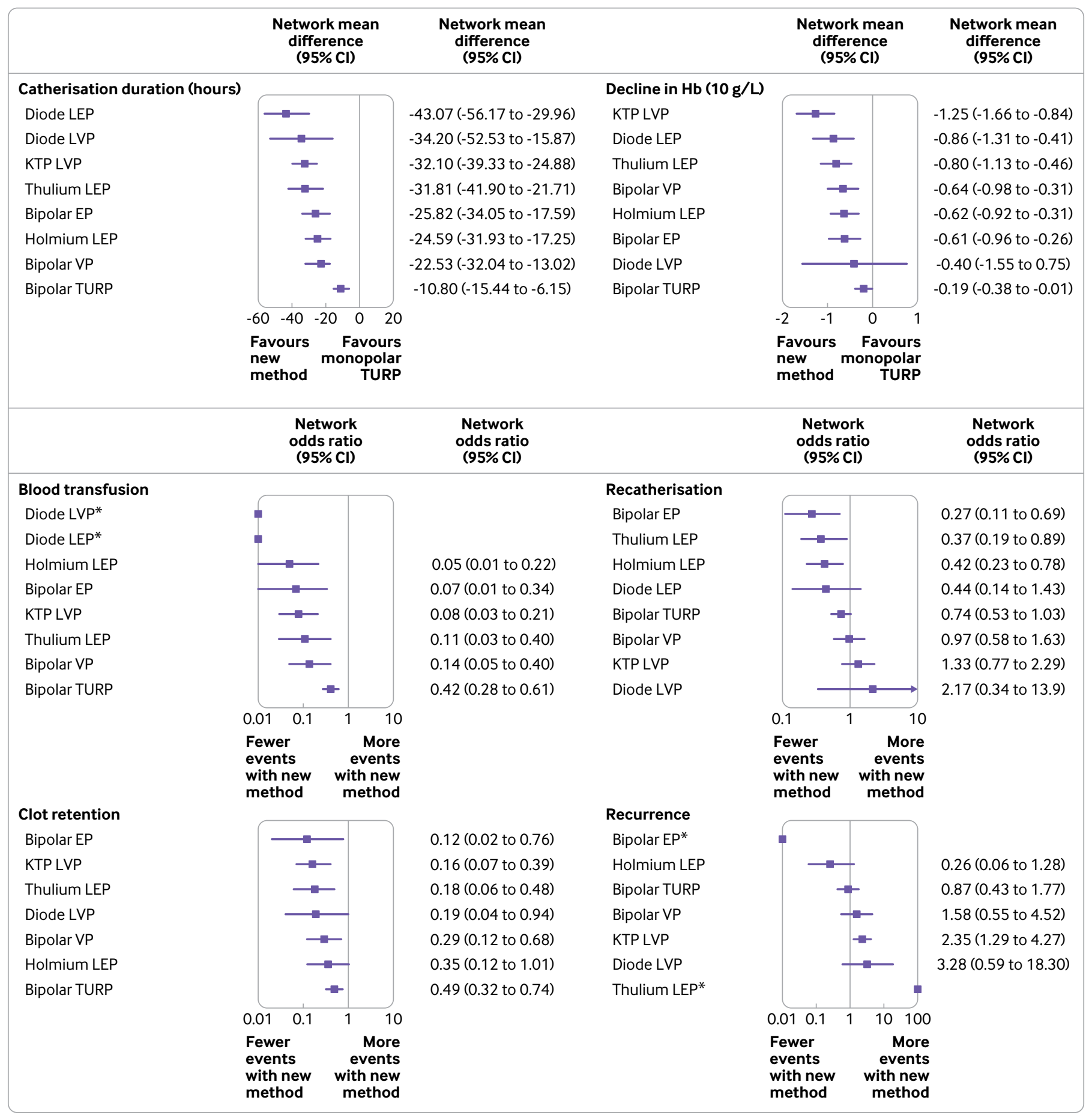

Fig 4 | Network meta-analysis of perioperative parameters and complications of new surgical methods compared with monopolar transurethral resection of the prostate for benign prostate hyperplasia. Common heterogeneity variables for all comparisons in this network meta-analysis included: $\mathrm{t}=0.39,12.3,0.56,0.05,0$, and 0 for haemoglobin declination, duration of catherisation, clot retention, blood transfusion, recatheterisation, and recurrence, respectively. Treatments ranked according to the SUCRA values. * 0 events in either new method or monopolar TURP groups. SUCRA=surface under the cumulative ranking; the unit for catheterisation duration and decline in haemoglobin is hours and $10 \mathrm{~g} / \mathrm{L}$, respectively. Abbreviations of surgical methods are listed in table 1 
for diode LVP. Recurrence was reported in 29 trials. Enucleation methods and bipolar TURP performed better than vapourisation methods (fig 4).

Retrograde ejaculation, urinary tract infection, incontinence, and stricture were reported in 17, 44, 50 , and 83 trials, respectively. We saw no significant difference between the new methods and monopolar TURP for these complications (supplementary fig 6 and supplementary table 5).

\section{Sensitivity analysis and inconsistency}

When grouping the nine methods into four groups, we found enucleation methods improved Qmax by $1.98 \mathrm{~mL} / \mathrm{s}$ (95\% confidence interval 0.55 to 3.41 ), $1.71 \mathrm{~mL} / \mathrm{s}$ (0.53 to 2.88 ), $4.12 \mathrm{~mL} / \mathrm{s}$ (1.11 to 7.12 ), and $4.82 \mathrm{~mL} / \mathrm{s}$ ( 0.96 to 8.68$)$ more than vapourisation methods at $6,12,24$, and 36 months after surgical treatment, respectively (table 2).

Regarding the prostate volume effect, the metaregression showed that the mean prostate volume moderated the treatment effect. In the large prostate group (mean prostate volume $>70 \mathrm{~mL}$ ), enucleation methods improved the Qmax values at six and 12 months after surgical treatment by $5.50 \mathrm{~mL} / \mathrm{s}(95 \%$ confidence interval 2.04 to 8.95 ) and $4.61 \mathrm{~mL} / \mathrm{s}$ (0.85 to 8.36) more than vapourisation methods, respectively. By contrast, for a mean prostate volume less than $70 \mathrm{~mL}$, enucleation only improved Qmax values by $0.45 \mathrm{~mL} / \mathrm{s}(-1.03$ to 1.93$)$ and $0.52 \mathrm{~mL} / \mathrm{s}$ $(-0.58$ to 1.62$)$ more than vapourisation at six and 12 months, respectively (supplementary table 10). Enucleation methods had more events of short term transient urinary incontinence than resection methods (odds ratio 1.92, 1.39 to 2.65). By contrast, permanent incontinence was rare regardless of the used method (for enucleation $v$ resection odds ratio $1.23,0.29$ to 5.22; supplementary fig 7).

We found no evidence of global inconsistency in any primary or secondary outcomes using the design-bytreatment interaction models except for catheterisation duration. After removing the single trial comparing bipolar TURP with thulium LEP, the inconsistency was no longer observed. No substantial inconsistency between direct and indirect comparisons was observed in the side-splitting models (supplementary table 9). Comparison-adjusted funnel plots also showed no small study bias (supplementary fig 4). Heterogeneity was high in various pairwise comparisons of functional outcomes and perioperative parameters. By contrast, we saw low heterogeneity in complications (supplementary table 5). The GRADE results and network comparison of post-voiding residual urine, IPSS quality of life, and hospital stay are shown in supplementary table 8 and supplementary figure 8 .

\section{Discussion \\ Principal finding}

Enucleation methods, including bipolar EP, holmium, thulium, and diode LEP, yielded greater Qmax values than resection and vapourisation methods at 6-12 months after surgical treatment. This difference could still be observed at 24-36 months after surgical treatment. Enucleation methods were more effective than vapourisation methods in large prostates. Enucleation methods also achieved better IPSS than resection and vapourisation methods, although the difference was not statistically significant. New methods were safer than monopolar TURP because blood transfusion, clot retention, haemoglobin decline, or transurethral resection syndrome were less likely. Our findings supported changes in the surgical treatment of benign prostatic hyperplasia from monopolar TURP to new methods.

Surgical treatment is usually performed when drug treatment fails to achieve satisfactory outcomes. Consequently, patients are older when surgical interventions are considered, leading to more comorbidities. $^{19} 20$ The new methods investigated here are therefore more suitable for these patients. The treatment goals for benign prostatic hyperplasia are not only to relieve lower urinary tract symptoms, but also to prevent adverse events related to benign prostatic hyperplasia, such as acute urinary retention, renal function deterioration, or bladder dysfunction. However, with the widespread use of drug treatments, the prevalence of adverse events related to benign prostatic hyperplasia has increased from 1998 to 2008. $^{2122}$ Additionally, Flanigan et al found that patients who underwent immediate TURP had greater improvements in Qmax and IPSS than those who were followed up with an extended period of watchful waiting, ${ }^{23}$ which seems to be a consequence of the

\begin{tabular}{|c|c|c|c|c|}
\hline \multirow[b]{2}{*}{ New surgical methods } & \multicolumn{4}{|c|}{ Time after surgery (months) } \\
\hline & 6 & 12 & 24 & 36 \\
\hline \multicolumn{5}{|c|}{ Qmax values (No of trials $=45,48,18$, and 14 , respectively) } \\
\hline Bipolar TURP & $0.66(-0.60$ to 1.92$)$ & $0.63(-0.16$ to 1.42$)$ & $-0.19(-2.14$ to 1.76$)$ & $0.06(-1.94$ to 2.05$)$ \\
\hline Enucleation & $1.52(0.36 \text { to } 2.69)^{*}$ & $1.49(0.59 \text { to } 2.40)^{\star}$ & $1.92(-0.22$ to 4.05$)$ & $2.85(0.13 \text { to } 5.57)^{\star}$ \\
\hline Vapourisation & $-0.44(-1.61$ to 0.73$)$ & $-0.21(-1.19$ to 0.76$)$ & $-2.20(-4.43$ to 0.31$)$ & $-1.98(-4.92$ to 0.97$)$ \\
\hline \multicolumn{5}{|c|}{ IPSS values (No of trials $=45,47,17,14$, respectively) } \\
\hline Bipolar TURP & $0.22(-0.58$ to 1.03$)$ & $-0.17(-0.72$ to 0.37$)$ & $-0.06(-0.92$ to 0.81$)$ & $-0.22(-1.11$ to 0.79$)$ \\
\hline Enucleation & $-0.17(-0.89$ to 0.54$)$ & $-0.84(-1.40 \text { to }-0.27)^{\star}$ & $-0.83(-1.79$ to 0.12$)$ & $-1.11(-2.27$ to 0.03$)$ \\
\hline Vapourisation & $0.52(-0.32$ to 1.37$)$ & $0.24(-0.45$ to 0.94$)$ & $1.12(-0.16$ to 2.41$)$ & $0.33(-1.03$ to 1.70$)$ \\
\hline
\end{tabular}


delay in effective treatment. With new surgical methods showing fewer complications but similar or even better effects than monopolar TURP, early surgical treatments might be considered to avoid adverse events related to benign prostatic hyperplasia.

Enucleation methods using fibreoptic lasers or bipolar loops mimic open prostatectomy. ${ }^{24}$ Thus, the fact that enucleation methods achieved the best Qmax values compared with resection and vapourisation methods is not surprising, because enucleation removes more tissue and results in greater reduction in prostate specific antigen than resection and vapourisation. ${ }^{25}$ In the medical treatment of benign prostatic hyperplasia, $\alpha$ blockers with or without $5 \alpha$-reductase inhibitors has been shown to improve Qmax by about 0.9-2.4 mL/s compared with placebo, which has been considered clinically significant. ${ }^{26} 27$ In our study, enucleation methods were found to improve Qmax by 1.71-1.98 $\mathrm{mL} / \mathrm{s}$ more than vapourisation methods, and by 4.12 and $4.82 \mathrm{~mL} / \mathrm{s}$ more at 6-12 and 24-36 months after surgical treatment, respectively. Hence, the difference in Qmax between enucleation and vapourisation methods was clinically meaningful. Our analysis also showed that vapourisation methods seemed to yield a higher recurrence rate of benign prostatic hyperplasia than enucleation or resection methods.

A previous meta-analysis of six randomised controlled trials with 541 patients found that holmium LEP achieved better Qmax values at 12 months after surgical treatment than monopolar TURP, although no differences in IPSS were found. ${ }^{28}$ Another metaanalysis comparing KTP LVP with monopolar TURP comprised six randomised controlled trials and five case-control studies involving a total of 889 patients $^{29}$ and found no difference in Qmax and IPSS when the prostate size was less than $70 \mathrm{~mL}$, but the Qmax and IPSS values in the KTP LVP group were lower for prostate sizes more than $70 \mathrm{~mL}$. Our results confirmed that enucleation methods performed better than resection methods when either bipolar or laser energy were used; however, the vapourisation method was unsuitable for large prostates.

\section{Complications}

No occurrence of transurethral resection syndrome associated with the eight new methods was reported. Regarding bleeding, our study showed that the eight new methods yielded better outcomes than monopolar TURP, both intraoperatively and postoperatively. Enucleation and vapourisation methods performed better than resection methods regardless of the energy system used. Vapourisation also produced coagulation effects, thereby leading to less bleeding. Only once during an enucleation procedure was a bleeding vessel encountered in the capsule region, compared with several times during resection procedures. This difference could have contributed to the decrease in blood loss associated with enucleation. With respect to postoperative bleeding, shorter catheterisation duration and fewer clot retention events were associated with less postoperative bleeding and better haemostatic effects. Laser energy, especially diodes and KTP, showed advantages over bipolar and monopolar energy in postoperative bleeding. Shorter catheterisation durations and fewer clot retention events could lead to a shorter hospital stay, reducing hospital costs and readmission rates.

Regarding the recatheterisation rate, enucleation methods were also better than resection, whereas vapourisation was the worst method. Enucleation methods remove more apical prostate tissue, whereas vapourisation methods remove less apical prostate tissue because of the risk of sphincter injury. ${ }^{30}$ Hence, some surgeons resect the apex of the prostate after vapourisation, to overcome the drawbacks of vapourisation. $^{31}$

Our study showed that enucleation methods yielded better functional outcomes and equivalent safety than vapourisation methods. However, the risk of short term transient incontinence was higher in enucleation than in resection methods. Liu et al compared bipolar EP with bipolar TURP and found that, after Foley removal, the incontinence rate was higher in enucleation than in resection methods at 24 hours, ${ }^{32}$ while no difference was found at two weeks after surgical treatment. Hence, some authors used vapo-enucleation or modified techniques to reduce the transient incontinence rate. ${ }^{33}$

Monopolar electrode or neodymium-doped yttrium aluminium garnet lasers have been used for vapourisation of the prostate. However, they have not been widely adopted because of the poorer long term results, reduced efficiency, or increased complications compared with monopolar TURP. ${ }^{30} 34$ Our results suggest that new vapourisation methods still achieve poorer Qmax or IPSS than enucleation and resection methods. However, these differences were mainly observed in large prostates. Moreover, the technique is easier to perform and causes less bleeding, especially when using KTP and diode lasers. Hence, vapourisation of the prostate using new energy systems is a promising technique for patients with smaller prostates or higher bleeding risks and those more suited for outpatient surgery. Some authors have tried to use a hybrid method (vapourisation with resection) to improve the efficacy of vapourisation. ${ }^{35}$ As high energy laser technology is evolving, the efficiency of vapourisation is expected to improve. Whether it can improve functional outcomes, especially in patients with large prostates, will require further research. ${ }^{36}$

Regarding bipolar energy, we evaluated bipolar enucleation, resection, and vapourisation simultaneously. The efficacy and complication rates were better with bipolar EP and bipolar TURP than with monopolar TURP. Compared with laser systems, the bipolar energy machine is multifunctional and the equipment and medical consumable materials are less expensive. ${ }^{37}$ Bipolar energy is a promising energy system for surgery for benign prostatic hyperplasia and is more useful in developing countries. The use of enucleation, resection, or vapourisation methods depends on the surgeon's personal preference and 
the patient's condition, accounting for factors such as prostate volume and comorbidities.

Urologists prefer using resection or enucleation methods to treat large prostates $(>70-80 \mathrm{~mL})$ but prefer using laser modalities or vapourisation methods in patients with increased bleeding risk. ${ }^{38}$ Moreover, financial considerations, such as whether the cost is fully reimbursed by insurance companies, the availability of required equipment, and surgeons' skill and experience, also affect the choice of surgical method. With shared decision making becoming the norm in clinical care, clinicians should discuss the benefits and risks of different surgical approaches with their patients.

\section{Strengths and limitations of the study}

The strength of our study is that we simultaneously compared nine different surgical treatments for benign prostatic hyperplasia using a network meta-analysis. We compared Qmax and IPSS values at 6, 12, 24, and 36 months after surgical treatment to evaluate the short to mid-term effects of different treatments. Additionally, we included randomised controlled trials without language restrictions to avoid bias.

Our network meta-analysis had some limitations. Firstly, complications were rare, and some trials reported zero events, resulting in a less precise estimation of pooled odds ratio. However, heterogeneity was low and also favoured new methods in pairwise comparisons. Secondly, functional outcomes were assessed blindly in only $14 \%$ of trials, which could have led to bias in favour of the new methods. In addition, heterogeneity was high in many comparisons of primary outcomes. Initial prostate volume, the degree of urodynamic obstruction, and surgeon experience could account for the high heterogeneity of functional outcomes. Thirdly, we did not analyse early postoperative urinary symptoms such as dysuria, urgency, or post-micturition pain. Although these symptoms affect short term patient satisfaction, they usually improve with drug treatment by two to three months after surgical treatment.

Fourthly, we did not differentiate vapo-enucleation from enucleation, because the definition and technique are not standardised. Hence, differences in outcomes between vapo-enucleation and enucleation or different enucleation methods require further investigation. Another limitation was that our review did not include some new methods for treating benign prostatic hyperplasia such as prostatic urethral lift, prostate artery embolisation, robot assisted simple prostatectomy, and water vapourisation. Urethrae lift and prostate artery embolisation are mainly used in patients not suitable for surgery or anaesthesia, whereas robotic simple prostatectomy is indicated for very large prostates. Water vapourisation was first introduced in 2016 and has not yet been compared with TURP in any randomised controlled trial. Because the target patient population of these new methods is different from that in our review, we therefore excluded these methods in our network meta-analysis. Finally, we used the mean prostate size of each article in our meta-regression on the relation between prostate sizes and treatment efficacy because we did not have individual patient data. This approach is prone to ecological bias and study level confounding.

\section{Conclusion}

Compared with monopolar TURP, eight new endoscopic surgical methods for benign prostatic hyperplasia were shown to be superior in safety. Enucleation methods showed better Qmax and IPSS after surgery than vapourisation and resection methods. The efficacy of vapourisation in large prostates requires further research for more evidence.

Contributors: All authors had full access to all of the data in the study and take responsibility for the integrity of the data and the accuracy of the data analysis. S-WH and Y-KT contributed to the study concept and design, data analysis and interpretation, and writing of the report. C-YT and C-ST contributed to the literature searches, extracted data, assessed risk of bias, and wrote the report. M-CS and Y-CY contributed to data analysis. K-LC and Y-SP contributed to data interpretation and provided intellectual enrichment to the report. All authors met the ICMJE criteria for authorship and agree with the manuscript results and conclusions. Y-KT is guarantor. The corresponding author attests that all listed authors meet authorship criteria and that no others meeting the criteria have been omitted.

Funding: The study was supported partly by a grant from the National Science Council in Taiwan (grant No 106-2314-B-002-098-MY3). The sponsors had no role in the study design; collection, analysis, and interpretation of data; writing of the report; and decision to submit the article for publication.

Competing interests: All authors have completed the ICMJE uniform disclosure form at www.icmje.org/coi_disclosure.pdf and declare: support from the National Science Council in Taiwan for the submitted work; no financial relationships with any organisations that might have an interest in the submitted work in the previous three years; no other relationships or activities that could appear to have influenced the submitted work.

Ethical approval: Not required.

Data sharing: No additional data available.

The lead author affirms that the manuscript is an honest, accurate, and transparent account of the study being reported; that no important aspects of the study have been omitted; and that any discrepancies from the study as planned (and, if relevant, registered) have been explained.

This is an Open Access article distributed in accordance with the Creative Commons Attribution Non Commercial (CC BY-NC 4.0) license, which permits others to distribute, remix, adapt, build upon this work non-commercially, and license their derivative works on different terms, provided the original work is properly cited and the use is noncommercial. See: http://creativecommons.org/licenses/by-nc/4.0/.

1 Chapple CR. Lower urinary tract symptoms suggestive of benign prostatic obstruction--Triumph: design and implementation. Eur Urol 2001;39(Suppl 3):31-6. doi:10.1159/000052565

2 Wei JT, Calhoun E, Jacobsen SJ. Urologic diseases in America project: benign prostatic hyperplasia. J Urol 2005;173:1256-61. doi:10.1097/01.ju.0000155709.37840.fe

3 Malaeb BS, Yu X, McBean AM, Elliott SP. National trends in surgical therapy for benign prostatic hyperplasia in the United States (2000-2008). Urology 2012;79:1111-6. doi:10.1016/j. urology.2011.11.084

4 Reich O, Seitz M, Gratzke C, Schlenker B, Walther S, Stief C. [Benign prostatic hyperplasia (BPH): Surgical therapy options]. Urologe A 2010;49:113-26. doi:10.1007/s00120-009-2183-1

5 Lourenco T, Pickard R, Vale L, et al, Benign Prostatic Enlargement team. Minimally invasive treatments for benign prostatic enlargement: systematic review of randomised controlled trials. BMJ 2008;337:a1662. doi:10.1136/bmj.a1662

6 Reich O, Gratzke C, Bachmann A, et al, Urology Section of the Bavarian Working Group for Quality Assurance. Morbidity, mortality and early outcome of transurethral resection of the prostate: a prospective multicenter evaluation of 10,654 patients. J Urol 2008;180:246-9. doi:10.1016/j.juro.2008.03.058 
7 Issa MM. Technological advances in transurethral resection of the prostate: bipolar versus monopolar TURP. J Endourol 2008;22:158795. doi:10.1089/end.2008.0192

8 Grande M, Facchini F, Moretti M, et al. [History of laser in BPH therapy]. Urologia 2014;81(Suppl 23):S38-42. doi:10.5301/ RU.2014.11985

9 Moher D, Liberati A, Tetzlaff J, Altman DG, PRISMA Group. Preferred reporting items for systematic reviews and meta-analyses: the PRISMA statement. Ann Intern Med 2009;151:264-9, W64. doi:10.7326/0003-4819-151-4-200908180-00135

10 Higgins J, Green S. Cochrane handbook for systematic reviews of interventions. The Cochrane Collaboration; 2011 [updated March 2011], version 5.1.0.

11 Puhan MA, Schünemann HJ, Murad MH, et al, GRADE Working Group. A GRADE Working Group approach for rating the quality of treatment effect estimates from network meta-analysis. BMJ 2014;349:95630. doi:10.1136/bmj.g5630

12 Follmann D, Elliott P, Suh I, Cutler J. Variance imputation for overviews of clinical trials with continuous response. I Clin Epidemiol 1992;45:769-73. doi:10.1016/0895-4356(92)90054-Q

13 White IR. Network meta-analysis. Stata / 2015:15:951-58. doi:10.1177/1536867X1501500403

14 Tu YK. Use of generalized linear mixed models for network meta-analysis. Med Decis Making 2014;34:911-8. doi:10.1177/0272989X14545789

15 White IR, Barrett JK, Jackson D, Higgins JP. Consistency and inconsistency in network meta-analysis: model estimation using multivariate meta-regression. Res Synth Methods 2012;3:111-25. doi:10.1002/jrsm.1045

16 Lu GB, Ades AE. Assessing evidence inconsistency in mixed treatment comparisons. J Am Stat Assoc 2006;101:447-59. doi:10.1198/016214505000001302

17 Salanti G, Ades AE, loannidis JP. Graphical methods and numerical summaries for presenting results from multiple-treatment metaanalysis: an overview and tutorial. J Clin Epidemiol 2011;64:163-71. doi:10.1016/j.jclinepi.2010.03.016

18 Mamoulakis C, Efthimiou I, Kazoulis S, Christoulakis I, Sofras F. The modified Clavien classification system: a standardized platform for reporting complications in transurethral resection of the prostate. World J Urol 2011;29:205-10. doi:10.1007/s00345-010-0566-y

19 Vela-Navarrete R, Gonzalez-Enguita C, Garcia-Cardoso JV, Manzarbeitia F, Sarasa-Corral JL, Granizo IJ. The impact of medical therapy on surgery for benign prostatic hyperplasia: a study comparing changes in a decade (1992-2002). BJU Int 2005;96:1045-8. doi:10.1111/j.1464-410X.2005.05735.x

20 Choi SY, Kim TH, Myung SC, et al. Impact of changing trends in medical therapy on surgery for benign prostatic hyperplasia over two decades. Korean J Urol 2012;53:23-8. doi:10.4111/ kju.2012.53.1.23

21 Stroup SP, Palazzi-Churas K, Kopp RP, Parsons JK. Trends in adverse events of benign prostatic hyperplasia (BPH) in the USA, 1998 to 2008. BJU Int 2012;109:84-7. doi:10.1111/j.1464 410X.2011.10250.x

22 Izard J, Nickel JC. Impact of medical therapy on transurethral resection of the prostate: two decades of change. BJU Int 2011;108:89-93. doi:10.1111/j.1464-410X.2010.09737.x

23 Flanigan RC, Reda DJ, Wasson JH, Anderson RJ, Abdellatif M, Bruskewitz RC. 5-year outcome of surgical resection and watchful waiting for men with moderately symptomatic benign prostatic hyperplasia: a Department of Veterans Affairs cooperative study. J Urol 1998;160:12-17. doi:10.1016/S0022-5347(01)63011-8

24 Kuntz RM, Ahyai S, Lehrich K, Fayad A. Transurethral holmium laser enucleation of the prostate versus transurethral electrocautery resection of the prostate: a randomized prospective trial in 200 patients. J Urol 2004;172:1012-6. doi:10.1097/01. ju.0000136218.11998.9e
25 Placer J, Gelabert-Mas A, Vallmanya F, et al. Holmium laser enucleation of prostate: outcome and complications of selftaught learning curve. Urology 2009;73:1042-8. doi:10.1016/i. urology.2008.12.052

26 Narayan P, Tewari A. A second phase III multicenter placebo controlled study of 2 dosages of modified release tamsulosin in patients with symptoms of benign prostatic hyperplasia. United States 93-01 Study Group. J Urol 1998;160:1701-6. doi:10.1016/ S0022-5347(01)62386-3

27 McConnell JD, Roehrborn CG, Bautista OM, et al, Medical Therapy of Prostatic Symptoms (MTOPS) Research Group. The long-term effect of doxazosin, finasteride, and combination therapy on the clinical progression of benign prostatic hyperplasia. N Engl) Med 2003;349:2387-98. doi:10.1056/NEJMoa030656

28 Yin L, Teng J, Huang CJ, Zhang X, Xu D. Holmium laser enucleation of the prostate versus transurethral resection of the prostate: a systematic review and meta-analysis of randomized controlled trials. J Endourol 2013;27:604-11. doi:10.1089/end.2012.0505

29 Ding H, Du W, Lu ZP, Zhai ZX, Wang HZ, Wang ZP. Photoselective green-light laser vaporisation vs. TURP for BPH: meta-analysis. Asian J Androl 2012;14:720-5. doi:10.1038/aja.2012.56

30 Gallucci M, Puppo P, Perachino M, et al. Transurethra electrovaporization of the prostate vs. transurethral resection. Results of a multicentric, randomized clinical study on 150 patients. Eur Urol 1998;33:359-64. doi:10.1159/000019616

31 Tefekli A, Muslumanoglu AY, Baykal M, Binbay M, Tas A, Altunrende $\mathrm{F}$. A hybrid technique using bipolar energy in transurethral prostate surgery: a prospective, randomized comparison. J Urol 2005;174:1339-43. doi:10.1097/01. ju.0000173075.62504.73

32 Liu JF, Liu CX, Tan ZH, Li SX, Li XZ, Chi N. [Transurethral bipolar plasmakinetic enucleation and resection versus transurethral bipolar plasmakinetic resection of the prostate for $\mathrm{BPH}$ : a randomized controlled trial on the incidence of postoperative urinary incontinence]. Zhonghua Nan Ke Xue 2014;20:165-8.

33 Takiuchi $\mathrm{H}$, Nakao A, Ihara $\mathrm{H}$. [Prevention of transient urinary incontinence in peri-operative period of modified holmium laser enucleation of the prostate (HoLEP)]. Hinyokika Kiyo 2008;54:475-8.

34 Hoekstra RJ, Van Melick HH, Kok ET, Ruud Bosch JL. A 10-year follow-up after transurethral resection of the prostate, contact laser prostatectomy and electrovaporization in men with benign prostatic hyperplasia; long-term results of a randomized controlled trial. BJU Int 2010:106:822-6 doi:10.1111/j.1464410X.2010.09229.x

35 Chau K, Yee C, Chan C, Chan E, Hou S, Ng C. A prospective randomized controlled trial comparing efficacy of hybrid bipolar transurethral vaporization and resection of prostate with bipolar transurethral resection of prostate (TURP). BJU Int 2014;113:13. https://doi.org/10.1111/bju.12606. https://www.cochranelibrary. com/central/doi/10.1002/central/CN-01011069/full

36 Ben-Zvi T, Hueber PA, Liberman D, Valdivieso R, Zorn KC. GreenLight XPS 180 W vs HPS 120 W laser therapy for benign prostate hyperplasia: a prospective comparative analysis after 200 cases in a single-center study. Urology 2013;81:853-8. doi:10.1016/j. urology.2012.12.031

37 Rai P, Srivastava A, Dhayal IR, Singh S. Comparison of safety, efficacy and cost effectiveness of photoselective vaporization with bipolar vaporization of prostate in benign prostatic hyperplasia. Curr Urol 2018;11:103-9. doi:10.1159/000447202

38 Gravas S, Cornu JN, Gacci M, et al. Management of Non-neurogenic Male LUTS 2019 [accessed 22 August 2019]. https://uroweb.org/ guideline/treatment-of-non-neurogenic-male-luts/

Web appendix 1: Study protocol

Web appendix 2: Supplementary materials 